\title{
IMPORTANCE OF IMPACTS ON FOREST ECOSYSTEMS FOR A SUSTAINABLE USE AND MAINTENANCE OF BIODIVERSITY
}

\author{
Harald Vacik ${ }^{*}$, Bernhard Wolfslehner*, Felix Heckl ${ }^{+}$and Josef Hackl ${ }^{+}$ \\ ${ }^{(*)}$ Institute of Silviculture, University of Agricultural Sciences, Vienna \\ A-1190 Vienna, Peter Jordanstr. 70, Austria \\ vacik@edv1.boku.ac.at \\ b.wolfslehner@gmx.at \\ ${ }^{(+)}$Federal Environment Agency Ltd - Austria \\ A - 1090 Vienna, Spittelauer Lände 5, Austria \\ heckl@ubavie.gv.at \\ hackl@ubavie.gv.at
}

Keywords: biodiversity, forest ecosystems, impacts, geometric mean, Analytical Hierarchy Process

\begin{abstract}
Summary: A study was carried out to describe and quantify the impacts on biological diversity in forest ecosystems and their working complexes not just of a sectoral point of view but with regards to an ecosystem approach. For this complex, multi-criteria decision problem the methodology of the Analytic Hierarchy Process (AHP) was incorporated. Structuring and systematizing aimed to identify all relevant impact factors, their sources and their qualitative and quantitative importance without considering the source of impact. Six main groups of impact factors could be identified, which had been further decomposed in a hierarchical structure. The impacts are provoked by several sources including forestry, agriculture, industries, trade, traffic, tourism, hunting and nature conservancy. The geometric mean is used to synthesize the individual judgments of 14 experts for each impact factor and for all impact sources. The problems with an operative definition of biodiversity, the lack of objective information on the importance of various impact factors on the sustainable use and maintenance of biodiversity in forest ecosystems and the generally known problem of the inaccuracy of the verbal comparison scale are discussed.
\end{abstract}

\section{Introduction}

Forest ecosystems are characterized by a complex of various elementary factors: plant, animal and microorganism communities, abiotic factors (climate, abiotic soil substance) and humanity as an integral component of ecosystems. Biodiversity means the variability among living organisms from all sources including, inter alia, terrestrial and other aquatic ecosystems and the ecological complexes of which they are part. This includes not only species richness as structural and process diversity within species, between species and of ecosystems is also a key component of biodiversity. According to the protocols of the UNCD Earth Summit at Rio de Janeiro and the Conferences on the Protection of European Forests at Helsinki (1993) and Lisabon (1998) biodiversity has been identified as a key issue for the sustainable use of forest resources. Therefore in forest management planning, emphasis is beeing placed not only on timber production but also on a sustainable use and maintenance of biodiversity.

Beside evolutionary processes it has been human use, settling and management which influences landscapes and (forest) ecosystems in an intensive and sustainable way. Due to increasing human use there is an ongoing trend to a shift from natural landscapes to land developed and cultivated by man.

Forest management affects various levels of biodiversity from the gene to the landscape (Franklin, 1993). 
In most European countries there is heavy human pressure on the forest and a correspondingly large human use of the multiple functions of the forest. On average there is less than 0.5 ha forest and other wooded land for each European and considerably less in densely populated urban countries. Losses of forest area to urban and transport infrastructure are compensated by afforestation of agricultural and other land, by about 1 million ha/year which leads to slowly expanding forests. In most european forest ecosystems changes of nutrient, water and energy cycles, genetic diversity, species composition, habitats and landscape structures can be observed. Forests are damaged by wildlife and grazing, insects, diseases and pollution. There is also a linkage between acid deposition and forest decline. Forest trees are influenced directly and indirectly through numerous effects like loss of soil bases, aluminium toxicity, interactions with air pollutants such as ozone and sulfur dioxide and interaction with natural phenomena such as drought and deficiencies of soil nutrients (Gorham, 1998).

The combination of direct and indirect actions of man on forests can contribute to a decrease in intraspecific variability, species diversity and ecosystem variety. The main sources for the multiple impacts are provoked by forestry, agriculture, industries, traffic and tourism. The understanding and evaluation of impacts of man on biodiversity is essential for asuring progressive improvement of forest ecosystems in that respect. Bearing in mind that each operation (e.g. harvesting, road construction, the use of herbicides and pesticides, doing meliorations and afforestations) and the effects of air pollution and global climate change has both positive and negative consequences for various living organisms and communities, this assessment plays a key role as basis for the promotion of sustainable forest management.

\section{Method}

Multiple criteria decision making (MCDM) methods are procedures and mathematical algorithms for aiding decision making when multiple objectives are considered. If a decision problem is characterized by a relatively small number of alternatives and the alternatives are represented in terms of attributes, it is called a multiple-attribute problem.

Assessing the impacts of various direct and indirect actions of man on forests which are provoked by different sources can be a wicked decision problem. In applying MCDM methods within the framework of assessing impacts for a sustainable use and maintenance of biodiversity the criteria are the different impact factors $Z_{1}, Z_{2}, Z_{3}, \ldots, Z_{k}$. These criteria take the form of discrete attributes and are not defined as continuous functional relationships. Instead, they take general qualitative forms $f_{i}=(i=1,2, \ldots, k)$ some of which are measurable directly or implicitly, while others are inherently qualitative and defy formal quantification.

For such complex, multi-criteria decision problems with both qualitative and quantitative aspects the methodology of the Analytic Hierarchy Process (AHP) can be incorporated. The AHP developed by Saaty $(1977,1980)$ is a robust and flexible multi-criteria decision analysis technique based on the prior articulation of preferences by the decision maker. In the context of the hierarchy described in figure 1 the MCDM problem may now be viewed as a process involving the determination of the relative importance of each impact factor and impact source relative to the higher levels of the hierarchy. This approach is used to arrive at a ratio-scale cardinal ranking of the impact sources.

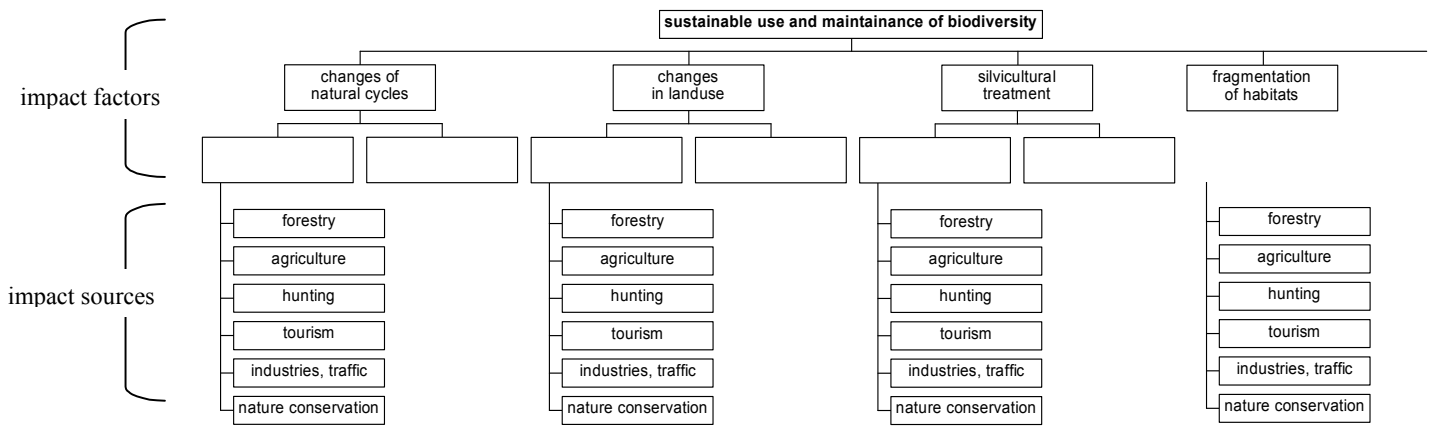

Fig. 1: structuring of the MCDM problem in a hierarchy of impact factors and impact sources 
Since its development, AHP has been applied in a wide variety of practical applications, including those related to economics and planning, conflict resolution, project selection and others. But applications in forestry are scarce (Howard, 1991, Kangas and Kuusopalo, 1993). The AHP can be summarized as a four-step procedure (Mollagashemi and Pet-Edwards, 1997; Mendoza and Prabhu, 2000):

1. Setting up of the decision hierarchy by decomposing the decision problem into a hierarchy of interrelated elements. Each level must be linked to the next-higher level and adjacent elements within one level must not be too disparate.

2. Generating input data consisting of comparative judgement by pairwise comparisons of decision elements.

3. Synthesizing the judgments and estimate the relative weights by using the "eigenvalue" method to generate a derived ratio scale that reflects the local priorities of the elements in the hierarchy.

4. Determination of the aggregate relative weights of the decision elements to arrive at a set of rating for the decision alternatives.

If there are more individual judgments $X_{i}(i=1-n)$ to aggregate, Aczel and Saaty (1983) provide a methode to derive a rational group choice based on four conditions:

- Separability condition $(S)$ means that the influences of the individual judgments $X_{i}$ can be separated

- Unanimity condition $(U)$ means that if all individuals give the same judgment $X$ that judgment should also be the synthesized judgment $X_{u}$

- Homogenity condition $(H)$ means that if all individuals judge a ratio $u$ times large as another ratio, then the synthesized judgment $X_{u}$ should also be $u$ times as large

- Power conditions $\left(P_{p}\right)$ means that for instance if the $i^{\text {th }}$ individual judges the lenght of a side of a square to be $m_{i}$, the synthesized judgement $X_{u}$ on the area of that square will be given by the square of the synthesized judgment on the lenght of its side.

Aczel and Saaty (1983) showed that only the geometric mean satisfies all these conditions which helps to determine a possible synthesizing function:

$$
\bar{X}_{G}=\sqrt[n]{X_{1} \cdot X_{2} \cdot X_{3} \cdot \ldots \cdot X_{n}} \quad \text { with } \quad\left(X_{i}>0\right)
$$

\section{A case study}

A study was carried out to describe and quantify the impacts on biological diversity in forest ecosystems and their working complexes not just of a sectoral point of view but with regards to an ecosystem approach (Heckl et al., 2001). The term ecosystem approach, as used in relation to the Convention on Biological Diversity (CBD), is a broad concept implying a holistic, integrated approach to the conservation and sustainable use of biodiversity. It takes into account all components of ecosystems (including humans) and their complex interactions as well as the interconnectedness of the ecosystems. It seeks to achieve a satisfactory balance between conservation and development (Korn et al, 1999).

For this complex, multi-criteria decision problem the methodology of the Analytic Hierarchy Process (AHP) was incorporated. Structuring and systematizing aimed to identify all relevant impact factors, their sources and their qualitative and quantitative importance without considering the source of impact. Six main groups of impact factors could be identified, which had been further decomposed in a hierarchical structure (figure 2): 
- Silvicultural treatment of the forests

- Changes of natural cycles in terms of water cycle, soil aggregation and degradation

- Changes in species spectrum and composition

- Measures to enhance biological diversity (dead wood, genetic conservation program, natural forest reserve, species conservation, tempering impacts of climate change)

- Changes of land use

- Division and fragmentation of habitats

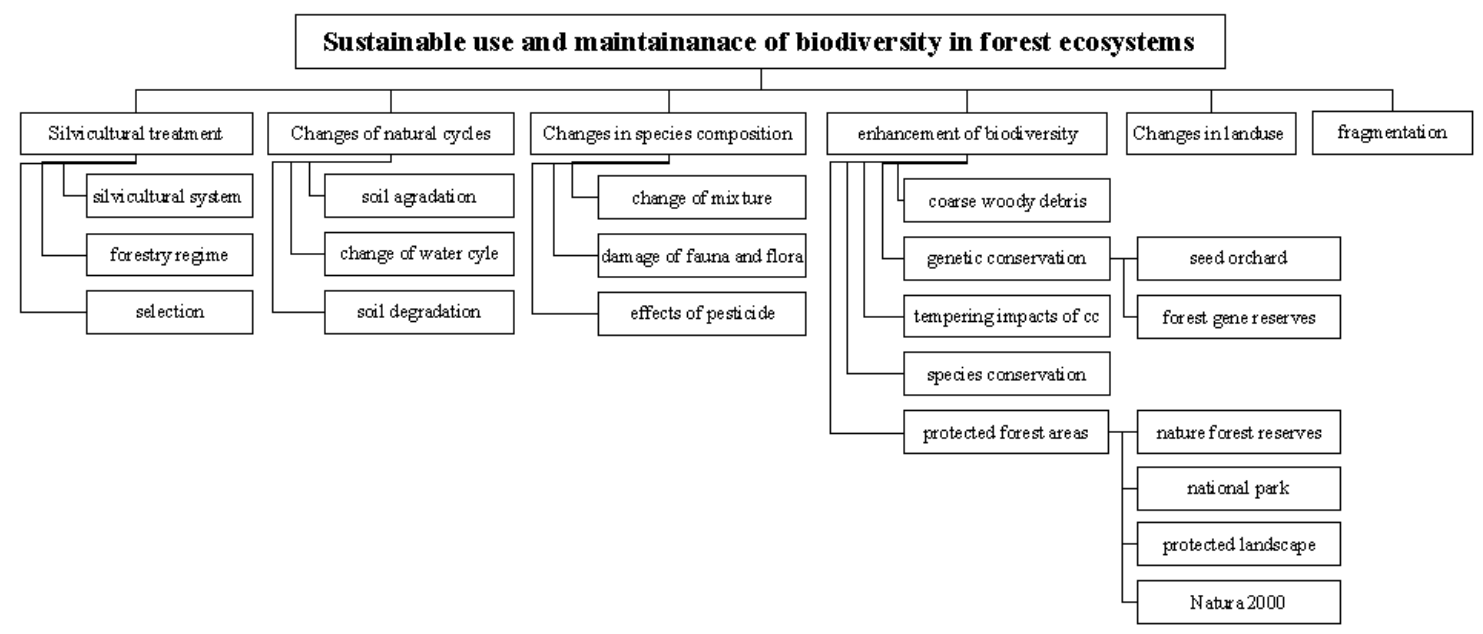

Fig. 2: Hierarchical structure of the impact factors $(\mathrm{n}=6)$ and decomposition in subordinate impact factors to judge their relevance for a sustainable use and maintenance of biodiversity in forest ecosystems

The impacts are provoked by several sources including forestry, agriculture, industries, trade, traffic, tourism, hunting and nature conservancy. These impacts are also of various importance at different spatial levels (global, national, bioregional, local) and at different levels of biological diversity (genetic, speciespopulations, ecosystems-habitats-landscapes). It is not possible to describe all effects of the most impacts on forest biodiversity by human landuse of forest ecosystems in quantitative terms and to consider all different levels. Therefore expert knowledge has to be utilized to assess the importance of different impacts in a qualitative way.

The mayor impacts can be described as follows:

- The impacts of forest practice and wood production like harvesting, afforestations, road construction, use of herbicides and pesticides, fertilications and meliorations, are mainly influencing species composition and structure of tree species and the ground vegetation.

- The impacts of agricultural landuse and production like nitrogen input by animal husbandry and fertilization and the rise of greenhouse gases (methane) influence water cycles of the forest ecosystems (irrigation). The reduction of landscape structures and number of plant species lead to a habitat change for wildlife animals.

- Air pollution from industries and transports has direct impacts on organisms and plays a mayor role in global climate change. Additionally the roads, railways and their effects (noise, waste, fragmentation) have severe impacts on habitats and landscapes.

- Leisure and sport activities in forests, touristic traffic and transport, touristic infra- and suprastructure can cause the removal of rare and endangered species.

- The traditional hunting practices and stock care, shooting plans and high stock numbers can influence the species composition.

- Through the pressure of nature conservancy groups natural forest reserves are established and species and habitat conservation programms are implemented. 
Interviews with experts from the science community, the government, private forest owners and NGO's allowed to derive the importance of the main impact sources (forestry, agriculture, industries, trade, traffic, tourism, hunting and nature conservation) for a sustainable use and maintenance of biodiversity in forest ecosystems through the formal analysis of the AHP. Experts were asked to determine the weights of different impacts on a sustainable use and production of biodiversity in forests by pairwise comparisons on each level of the hierarchy. At the last level of the hierarchy experts had to determine the importance of forestry, agriculture, industries, trade, traffic, tourism, hunting and nature conservation for a particular impact like road building or use of pesticides. The experts should not assess the direction of the different impacts (positive or negative effects) on the diversity of forest ecosystems but the importance to influence the diversity.

Insights gained from this analysis method can be used in two ways:

(1) prioritization of impact factors and impact sources in terms of their significance to overall sustainable use and maintenance of biodiversity in forest ecosystems and

(2) prioritization of criteria under each impact factor

These prioritized lists could be used to guide policy decisions in relative to what impact factor and/or criteria are considered significant. In the light of limited resources (financial, time, personal) there could be a focus on those criteria that are considered most relevant and where the state of the actual forest ecosystem is lacking. 


\section{Results}

All individual judgments $(n=14)$ of the experts where used to derive a synthesized judgment for each impact factor and for all impact sources. Figure 3 indicates the synthesized judgments of all experts $(n=$ 14) for the importance of impact sources for the sustainable use and maintenance of biodiversity. Regarding the impact sources the results of the interviews show that forestry plays a major role in influencing biological diversity in forests due to the quantity and relation of its measures followed by impacts of the industrial sector and traffics. The other impact sources do not indicate a mayor relevance, whenever the variance of judgments is differing.

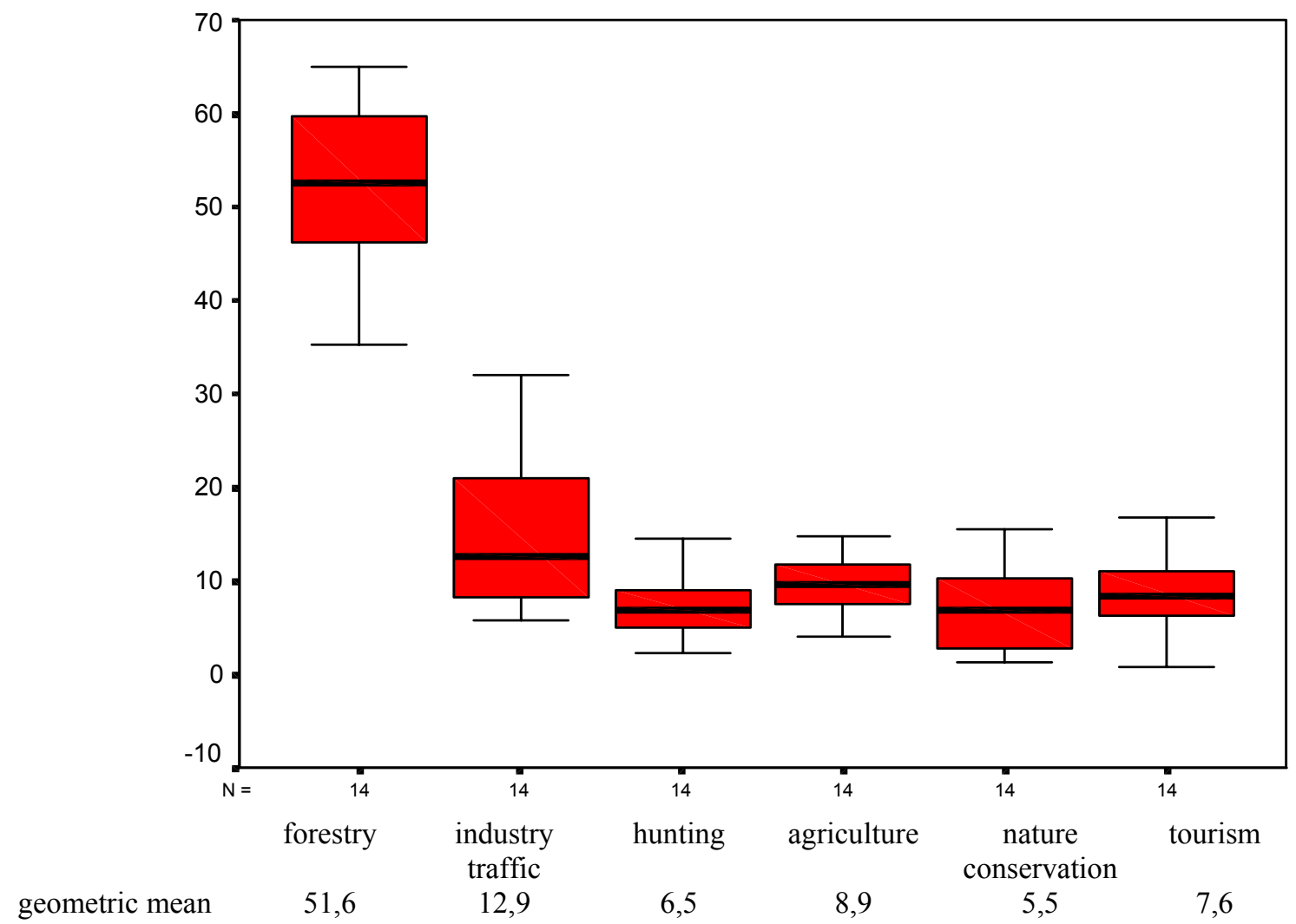

Fig. 3: Boxplot and geometric mean of the synthesized individual judgments of all experts $(n=14)$ for the importance of impact sources for the sustainable use and maintenance of biodiversity

The prioritization of impact factors in terms of their significance to overall sustainable use and maintenance of biodiversity in forest ecosystems and show similary results (Figure 4). The silvicultural treatment and various measures to enhance biodiversity (e.g. establishment of protected areas, management of coarse woody debris, species and genetic conservation programs) have been determined mostly relevant. The other main impact factors indicate a relative small importance. 


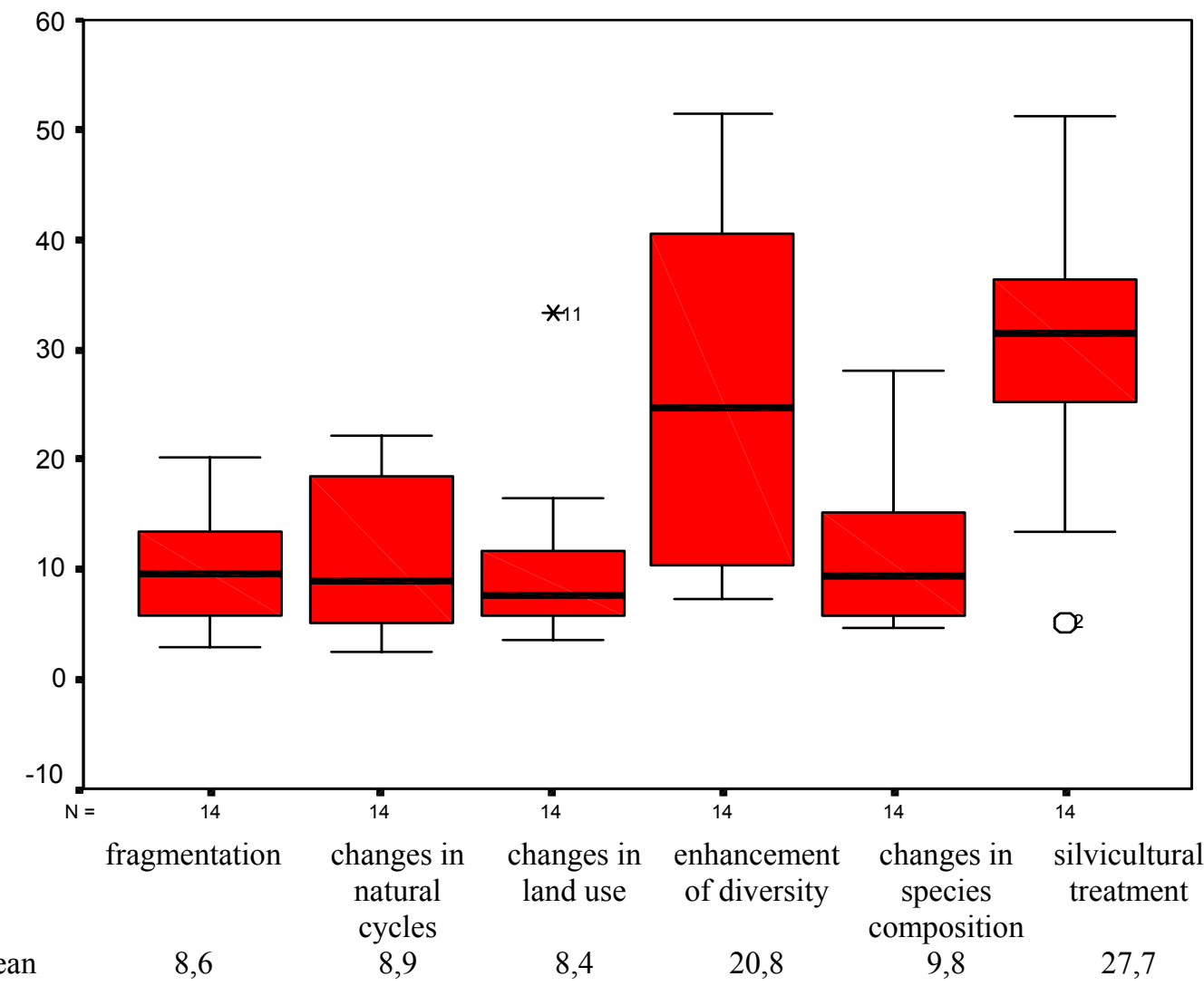

geometric mean

8,6

8,4

20,8

27,7

Fig. 4: Boxplot and geometric mean of the synthesized individual judgments of all experts $(n=14)$ for the importance of impact facts for the sustainable use and maintenance of biodiversity

Figure 5 indicates the relative proportion of impact sources for the main impact factors based on the geometric mean of the synthesized judgments of all experts. Again the experts considered forestry as the most important impact source, but the relative proportion varies between different impact factors.

The changes in species composition is mainly based on the effects of hunting practices (damages of flora by browsing and bark beeling of deer) and forestry (selection, choose of the silvicultural system and the forestry regime).

The engagement of nature conservation groups concerning species and habitat conservation, conservation of natural processes and legal frameworks (e.g. Natura 2000) are a driving forces in the sustainable use and protection of biodiversity in forest ecosystems. Therefore the experts have considered activities of nature conservation groups as the main impact source for the enhancement of biodiversity beside forestry. Concerning fragmentation and their negative effects on habitat and landscape the main impacts on forest ecosystems are coming from tourism, industries and traffic.

The changes of land use and natural cycles are mostly a consequence of the effects from agriculture, tourism, industries and traffic. Nitrogen input by animal husbandry and fertilization, formation of greenhouse gases (methane) and influences on water cycle and level are impacts coming from agriculture. Tourism, industries and traffic cause air pollution with direct impacts on organisms and via a global climate change and also infrastructural constructions (e.g. roads, railway) and their effects (e.g. noise, dust). Additonaly the leisure and sport activities in forests, touristic traffic and transport, touristic infraand suprastructure, waste and the removal of rare and endangered species are potential impacts on forest ecosystems. 


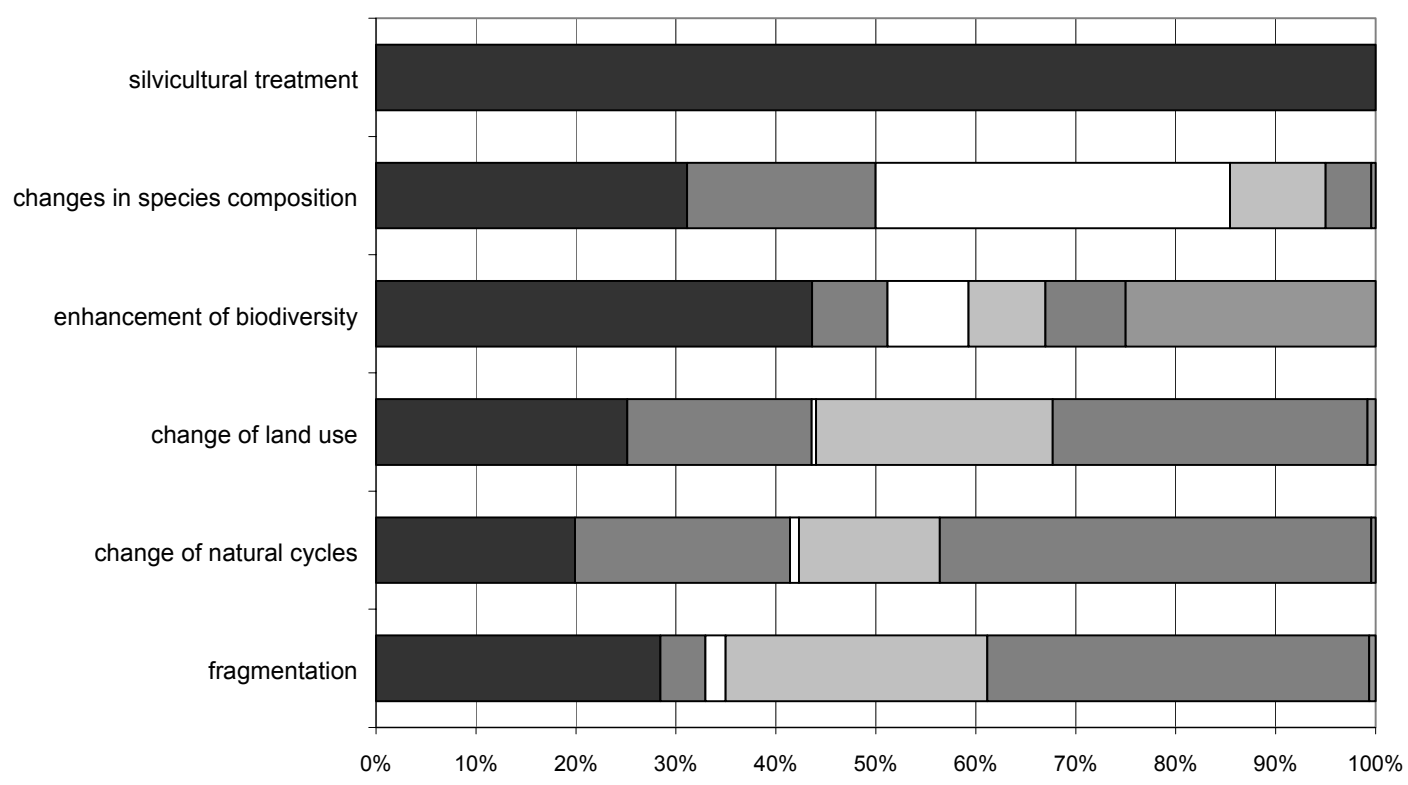

$\square$ forestry $\square$ agriculture $\square$ hunting $\square$ tourism $\square$ industries, traffic $\square$ nature conservation

Fig. 5: Relative proportion of impact sources for the main impact factors based on the geometric mean of the synthesized judgments of all experts $(n=14)$

\section{Discussion}

Sensitivity analysis was used to assess the importance of weighting of different impact factors and to identify the different level of perception of experts and decision makers. In interpretation of the results organizational aspects of the study and the different knowledge of experts in the various fields of forest ecosystem management has to be considered. The different impacts on a sustainable use and maintenance of biodiversity could be categorized in four groups:

- impacts where the effects on the diversity of forest ecosystems are well known and judgments can be done easyly (e.g. effects and importance of. a forest gene reserve)

- impacts where the effects on forest ecosystems are well known but not the effects on the diversity of forests (e.g. effects of pesticides and biocides). Therefore the experts have to judge the importance in a deductive way. The individual knowledge of each expert helps to determine the importance qualitativly.

- impacts where the effects on ecosystems are known, but there relevance for forest ecosystems or the diversity of forests are not studied yet (e.g. change of the land use). Therefore the experts have to judge the importance twice in a deductive way, the assessment becomes difficult.

- impacts where future effects are not well known and/or only scenarios are the basis for the judgements (e.g. effects of global climate change). In this case the value system, paradigms and individual preferences of the experts are mostly relevant.

A lot of scientific research activities in the thematic field diversity of forest ecosystems have been carried out in the last years, but for many cases detailled results are missing. The difficulties in determinating the relative importance of an impact factor increase with the lack of information. The experts maybe try to cover their uncertainties in balancing the values of the individual pairwise judgments. Additonally in some cases the variance of the individual judgments is massive. These outcomes may be interpreted as hints for existing knowledge gaps in science and practice and may lead to further scientific research 
activities in some areas. Especially the concentration of scientific research programs on the flora of forest ecosystems is evident.

Difficulties arised from the reduction of the complexity of the term biodiversity. Within the hierarchical structure the spatial (local, national, global), thematic (Species richness, patchiness, genetic diversity) and temporal aspects of biodiversity have not been considered. During some assessments in the beginning of the project it showed out, that none of the experts can spend the amount of time to do such detailed judgments. This reduction led to misunderstandings in deriving the judgments based on the presented hierarchical structure (figure 2). Through an intensive help during the assessments these problems could be solved.

The study showed the problems with an operative definition of biodiversity, the lack of objective information on the importance of various impact factors on the sustainable use and maintenance of biodiversity in forest ecosystems and the generally known problem of the inaccuracy of the verbal comparison scale are discussed. However, these prioritized lists helps to guide policy decisions in relative to what impact factor are considered significant. In the light of limited resources (financial, time, personal) there could be a focus on those criteria that are considered most relevant and where the state of the actual forest ecosystem is lacking. The pressure caused by the competing uses of land and the growing demand for services from forests call for careful intersectoral decision making in forest management and sustainable use of biodiversity.

\section{Acknowledgement}

This study was founded by the Ministery of Agriculture and Forestry, Environment and Water (Nr. 25 4496/14-II/5/99). We like to thank all the experts who took part in this study and gave us usefull comments on the hierarchical structure.

\section{References}

Aczèl, J. and Saaty, T.L. (1983) Procedures for Synthesizing Ratio Judgements, Journal of Mathematical Psychology, 27, 1, $113-122$.

Franklin, J.F. (1993) Preserving biodiversity: species, ecosystems or landscapes? Ecol. Appl. 3, 202-205. Gorham, E. (1998) Acid deposition and its ecological effects: a brief history of research. Environmental Science \& Policy, 1, 153-166.

Heckl, F., Hackl, J., Vacik, H. and Wolfslehner, B. (2001) Schutz und nachhaltige Nutzung der biologischen Vielfalt im Wald, UBA report, Federal Environment Agency Ltd, Austria (in print) Howard, A.F. (1991) A critical look at multiple criteria decision making techniques with reference to forestry applications. an, J.For.Res., 21, 1649-1659.

Kangas, J. and Kuusopalo, J. (1993) Integrating Biodiversity into Forest Management Planning and Decision Making, For. Ecol. Manage., 61, 1-15.

Korn, H., Stadler, J., Maltby, E. and Kerr A.J. [eds.] (1999) Report of the scientific workshop on "The ecosystem approach - what does it mean for European ecosystems?". BfN Skripten 9, German Federal Agency for Nature Conservation 1999.

Mendoza, G.A. and Prabhu, R. (2000) Multiple criteria decision making approaches to assessing forest sustainability using criteria and indicators: a case study. Forest Ecol. Manage., 131, 107-126.

Mollaghasemi, M. and Pet-Edwards, J., (1997) Technical briefing: making multiple-objective decisions. IEEE Computer Society Press, Los Alamitos.

Saaty, T.L. (1977) A scaling method for priorities in Hierarchical Structures. J.Math.Psychology 15, 234281.

Saaty, T.L. (1980) The Analytic Hierarchy Process, McGraw-Hill, New York. 
